\title{
Crucial role of plasmacytoid dendritic cells in the development of acute colitis through the regulation of intestinal inflammation
}

\author{
K Arimura $^{1,2}$, H Takagi ${ }^{1,3}$, T Uto ${ }^{1,3}$, T Fukaya ${ }^{1,3}$, T Nakamura ${ }^{1,4}, \mathrm{~N}_{\text {Choijookhuu }}{ }^{5}$, Y Hishikawa ${ }^{5}$, \\ Y Yamashita ${ }^{2}$ and K Sato ${ }^{1,3}$
}

Disruption of intestinal homeostasis can lead to inflammatory bowel diseases endowed susceptibility genes and environmental factors affecting intestinal accumulation and activation of colitogenic phagocytes. Plasmacytoid dendritic cells ( $\mathrm{pDCs}$ ) are immune cells that had been proposed to control innate and adaptive immunity through the massive secretion of type I interferon (IFN-I). However, the contribution of pDCs to the progression of intestinal inflammation remains unclear. Here we show a critical role of $\mathrm{pDC}$ in the initiation of acute colonic inflammation using T-cell-independent acute colitis model with a selective ablation of pDCs. Although pDCs accumulated in the inflamed colon upon mucosal injury, deficiency of pDCs attenuated the development of acute colitis independent of IFN-I signaling, accompanied by the diminished colonic production of proinflammatory cytokines. Furthermore, deficiency of pDCs impaired the mobilization of colitogenic phagocytes into the inflamed colon possibly mediated by the abrogated mucosal production of $\mathrm{C}-\mathrm{C}$ chemokine receptor 2 ligand. Thus, our findings highlight a critical role of pDCs in the induction of the colonic inflammation that regulates the colonic accumulation of inflammatory phagocytes leading to the initiation and exacerbation of acute colitis, and they may serve a key role in controlling gut mucosal immune homeostasis.

\section{INTRODUCTION}

Dendritic cells (DCs) are essential antigen (Ag)-presenting cells that have critical roles in orchestrating immune responses, linking innate information mediated by recognizing invading pathogens through a variety of pattern recognition receptors (PRRs), such as Toll-like receptors (TLRs), to tailored adaptive responses. ${ }^{1-3} \mathrm{DCs}$ are represented by two major lineages, classical or conventional DCs (cDCs) and plasmacytoid DCs (pDCs). ${ }^{1-3}$ cDCs have outstanding capacity to prime naive $\mathrm{T}$ cells to generate various types of effector $\mathrm{T}\left(\mathrm{T}_{\text {eff }}\right)$ cells owing to the prominent expression of major histocompatibility complex class (MHC) II and costimulatory molecues. ${ }^{4-6}$ On the other hand, pDCs are specialized in endosomal TLR7/9-mediated recognition of viral nucleic acids (NAs) and respond with the massive secretion of type I interferon (IFN-I). ${ }^{7-9}$ Therefore, pDCs have been considered as important mediators of antiviral responses. ${ }^{7-9}$ Furthermore, recent studies have suggested that the activation of pDCs by recognition of self-NA causes elevated IFN-I levels, ${ }^{10}$ and this process contributes to the induction of pathogenesis in psoriasis ${ }^{11}$ and systemic lupus erythematosus (SLE). ${ }^{12-14}$

The intestine is the largest compartment of the immune system that is lined by a single layer of epithelium harboring commensal bacteria. ${ }^{15,16}$ The gut microbiota contains $10^{14}$ microorganisms, including at least $10^{3}$ different species. ${ }^{15,16}$ The intestinal immune system has to balance protective immune responses to potentially pathogenic microorganisms with nonresponsiveness to commensal bacteria and dietary Ags to maintain immune homeostasis in this environment. ${ }^{17,18}$

Breakdown of intestinal homeostasis can result in inflammatory bowel diseases (IBDs), represented by Crohn disease

${ }^{1}$ Division of Immunology, Department of Infectious Diseases, Faculty of Medicine, University of Miyazaki, Miyazaki, Japan. ${ }^{2}$ Department of Oral and Maxillofacial Surgery, Faculty of Medicine, University of Miyazaki, Miyazaki, Japan. ${ }^{3}$ Japan Agency for Medical Research and Development (AMED), Tokyo, Japan. ${ }^{4}$ Department of Otolaryngology, Head and Neck Surgery, Faculty of Medicine, University of Miyazaki, Miyazaki, Japan and ${ }^{5}$ Division of Histochemistry and Cell Biology, Department of Anatomy, Faculty of Medicine, University of Miyazaki, Miyazaki, Japan. Correspondence: K Sato (katsuaki_sato@med.miyazaki-u.ac.jp). 
(CD) and ulcerative colitis (UC) in humans. ${ }^{19-22}$ Clinical and experimental evidence suggests that the etiology of IBD is complex and multifactorial, involving susceptibility genes and environmental factors, such as commensal microflora and their products as well as fed Ags, and their interaction leads to aberrant or dysregulated intestinal inflammatory immune responses. ${ }^{19-22}$ IBD displays chronic symptoms of abdominal pain, diarrhea, and bleeding in the gastrointestinal tract, in which $\mathrm{CD}$ primarily affects the small intestine and colon, whereas UC affects only the colon. ${ }^{21}$ A number of studies have been instrumental in defining the fundamental immunological aspects that lead to colonic inflammation in different models of experimental colitis, including acute colitis induced by a single cycle of dextran sodium sulfate (DSS) administration, which is believed to cause the destruction of gut epithelial cells (ECs) of the basal crypts and affect the integrity of the mucosal barrier, resulting in the translocation of luminal microbiota into the lamina propria (LP). ${ }^{23-25}$ It has been shown that DSS-induced chronic colitis can be mediated by several subsets of intestinal innate phagocytic cells mobilized into the inflamed colon, such as monocytes/macrophages and cDCs, to sense pathogenassociated molecular patterns of the enteric bacterial flora and damage-associated molecular pattern molecules released from host dying cells through a variety of PRRs, and the occurrence of the intestinal pathogenesis is independently of $\mathrm{T}$ cells. ${ }^{26-28}$ On the other hand, previous studies have shown the lack of peripheral blood pDCs in patients with active IBD, and instead their accumulation in the inflamed colonic mucosa and mesenteric lymph node (MLN) and dysfunction. ${ }^{29,30}$ However, how pDCs contribute to the development of IBD remains unclear.

To address the role of pDCs in the control of immune responses in vivo, we have previously generated Siglec-Hdiphtheria toxin (DT) receptor (DTR) knock-in mice, which harbors the expression of DTR under the control of the Siglech gene, allowing almost complete depletion of Siglec- $\mathrm{H}^{+}$pDCs in the lymphoid tissue by DT treatment (referred to as pDCablated mice), and demonstrated that pDCs have an important role in the control of inflammation and T-cell response as well as initiation and exacerbation of IFN-I-associated autoimmune diseases. ${ }^{31,32}$

In this study, we report that pDCs are requisite for the development of DSS-induced colitis using pDC-ablated mice. We further demonstrate that the accumulation of pDCs into LP has a crucial role in the recruitment of inflammatory monocytes/macrophage and cDCs into the inflamed colon, and that amplify the intestinal inflammation. Thus, our findings define a crucial role of pDCs in the development of acute colitis through the control of intestinal inflammatory responses.

\section{RESULTS}

pDC exacerbates acute colitis induced by a single-cycle administration of DSS

In line with our previous reports, ${ }^{31,32} \mathrm{pDC}$-ablated mice that express DTR under the control of the Siglech gene showed almost complete and specific elimination of $\mathrm{CD} 11 \mathrm{c}^{\mathrm{int}} \mathrm{BST} 2^{+}$ pDCs, which expressed B220 but not CD11b, among CD45.2 $2^{+}$ CD64 ${ }^{-}$leukocytes in the spleen (Spl) and MLN as well as colonic LP after single DT injection (Figure 1; data not shown). On the other hand, pDC-ablated mice had normal frequency of CD11 $\mathrm{c}^{\text {high }} \mathrm{BST}_{2}{ }^{-}$cDCs in these tissues compared with wildtype (WT) mice (Figure 1).

To address the role of pDCs in the initiation and progression of acute colitis, pDC-ablated mice were given 2\% DSS in drinking water for 7 day, and disease progression was daily monitored. After DSS treatment, WT mice manifested as a clinical symptom of acute colitis, including body weight (BW) loss and the disease activity index (DAI) marked by diarrhea and rectal bleeding as well as colonic atrophy (Figure 2a-d). On the other hand, pDC-ablated mice exhibited the ameliorated clinical symptom of acute colitis as compared with WT mice (Figure 2a-d), whereas the reconstitution of pDC-ablated mice with WT pDCs restored this pathogenesis (Figure $\mathbf{2} \mathbf{e}-\mathbf{h}$ and Supplementary Figures S1a,b online). Furthermore, pDCablated mice were more resistant to lethality induced by acute colitis than WT mice, and this lethality was correlated with the less clinical symptom during 14 days after initiation of DSS application (Supplementary Figure S1c,d).

We also examined the contribution of $\mathrm{cDCs}$ in the development of DSS-induced acute colitis using CD11cDTR/enhanced green fluorescent protein (EGFP) transgenic (Tg) mice, ${ }^{33}$ in which $\mathrm{CD} 11 \mathrm{c}^{\text {high }} \mathrm{BST} 2^{-}$cDCs, but not $\mathrm{CD} 11 \mathrm{c}^{\text {int }} \mathrm{BST}_{2}{ }^{+}$pDCs, were depleted in Spl, MLN, and colonic LP after single DT injection (referred to as cDCablated mice; Supplementary Figure S1e). Similar to the published report, ${ }^{26} \mathrm{cDC}$-ablated mice showed less symptom of acute colitis induced by a single cycle of DSS administration than WT mice, whereas there was no significant difference in the attenuation of the development of acute colitis between cDC-ablated and pDC-ablated mice (Supplementary Figure S1f,g).

Collectively, these results indicate that the deficiency of pDCs as well as CDCs attenuates the initiation and progression of DSS-induced acute colitis.

\section{pDCs accelerate DSS-induced colonic inflammation}

To clarify how pDCs contribute to the development of DSSinduced acute colitis, we examined the colonic pathological changes after oral DSS administration. Histological analysis of the sections from large intestine showed that DSS treatment caused colonic inflammation and tissue damage, including EC injury, mucosal ulceration, and massive leukocytic infiltration in WT mice. In contrast, pDC-ablated mice showed a lower pathological severity than WT mice following DSS application (Figure 3a,b).

To further evaluate colonic inflammation caused by DSS treatment, we analyzed the production of proinflammatory cytokines in colonic explants from DSS-treated mice. Colonic explants from DSS-treated WT exhibited the production of tumor necrosis factor- $\alpha$, interleukin-6 (IL-6), IL-12p40, and IFN $-\gamma$, whereas the production of these proinflammatory 
cytokines was markedly reduced in those from pDC-ablated mice when compared with WT mice (Figure 3c).

Taken together, these results indicate that the deficiency of pDCs improves DSS-induced colonic tissue disruption and inflammation.

As pDCs have shown to control certain immune responses through the production of IFN-I, ${ }^{7-14}$ we assessed the contribution of the IFN-I signaling to the control of the development of acute DSS-induced colitis using Ifnar $^{-/-}$ mice, which lacking $\operatorname{IFN}-\alpha / \beta$ receptor $\mathrm{I}$ (IFNAR1). ${ }^{34}$ Unexpectively, there was no substantial difference in the BW loss between WT and Ifnar $1^{-/-}$mice following oral DSS administration (Supplementary Figure S2a). Furthermore, the disease progression was also comparable between pDC-ablated and Ifnar $1^{-/}$pDC-ablated mice (Supplementary Figure S2b). Although oral administration of DSS enhanced the transcriptional expression of Ifn $b$ alone in colonic explants from WT mice, similar transcriptional expression levels of Ifna and Ifnb were observed in those from both DSS-fed WT and pDC-ablated mice (Supplementary Figure S2c).

Collectively, these results indicate that the regulation of DSSinduced colonic inflammation in the absence of pDCs is not affected by the deficency of IFNAR1.
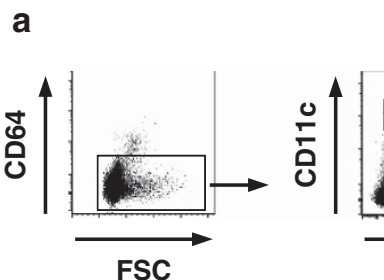

WT

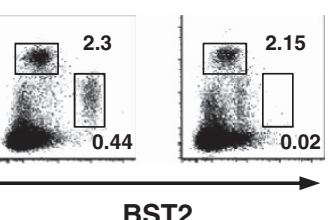

BST2
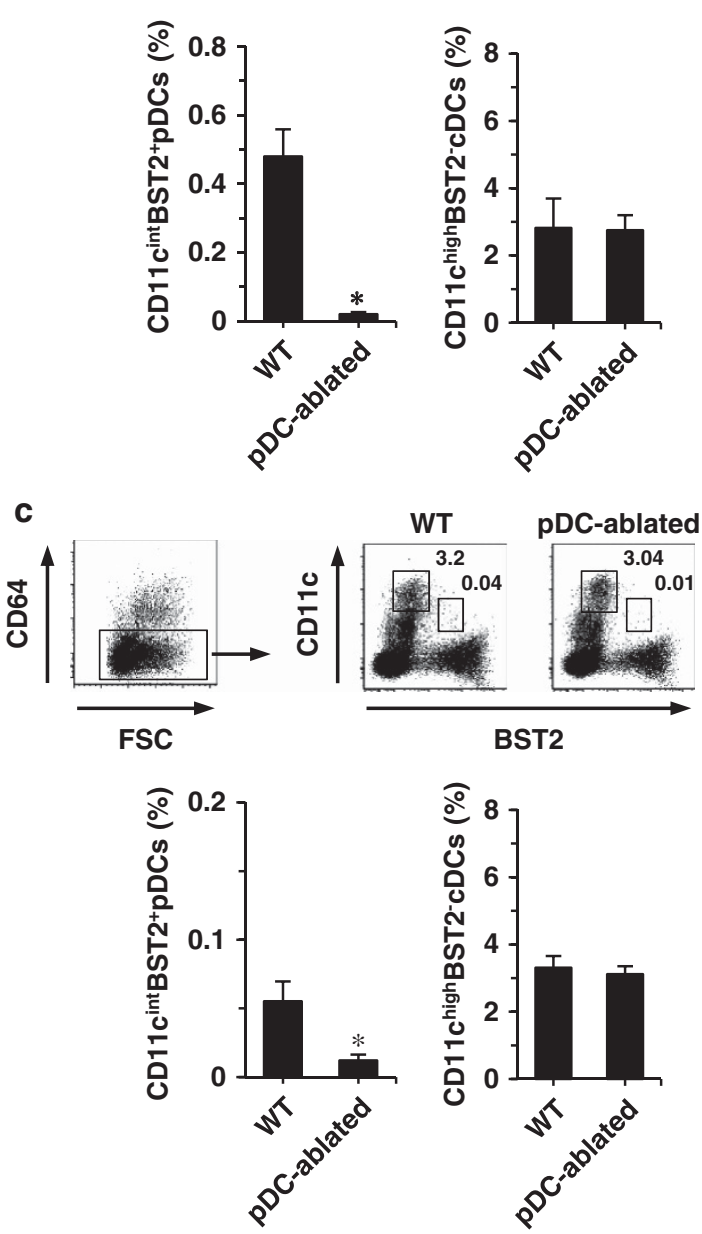

b

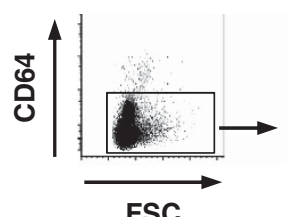

FSC
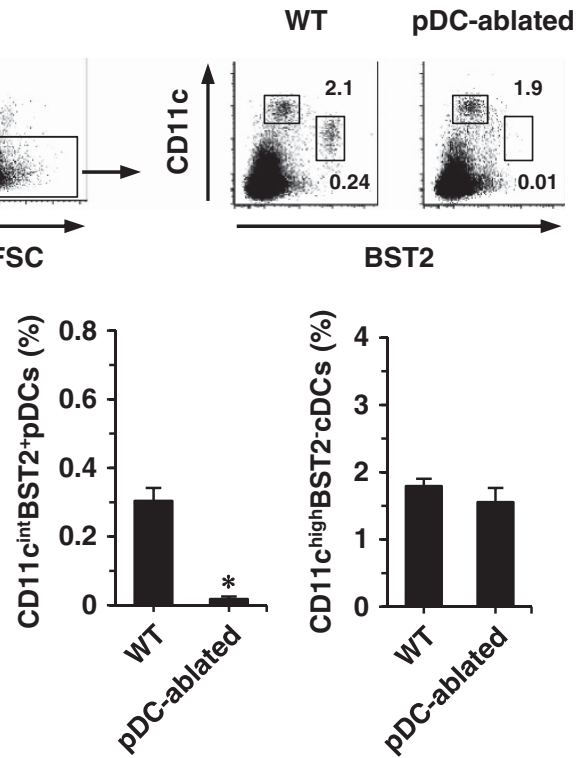

Figure 1 Specific elimination of pDCs in pDC-ablated mice. The frequency of CD11 $c^{\text {int }} B S T 2{ }^{+} p D C s$ and CD11c ${ }^{\text {high }} B S T 2^{-} c D C s$ in $S p l(a), M^{-} N(\mathbf{b})$, and colonic LP (c) obtained from the WT $(n=3)$ and pDC-ablated mice $(n=3)$ that had been treated with DT were analyzed by flow cytometry on 1 day after DT treatment. Data are presented as a dot plot, and numbers represent the proportion of the indicated cell populations among CD45.2 ${ }^{+}$CD64 ${ }^{-}$ leukocytes in each quadrant (upper panel). Data are the mean \pm s.d. from three individual samples in a single experiment (lower panel). ${ }^{\star} P<0.01$ compared with WT mice. All data are representative at least three independent experiments. DT, diphtheria toxin; MLN, mesenteric lymph node; pDC, plasmacytoid dendritic cell; Spl, spleen; WT, wild type. 
pDCs accumulate in the inflamed colonic LP after DSS treatment

As inflammatory phagocytic cells, such as monocytes/macrophages and cDCs, have been shown to mobilize into the inflamed colon in the development of acute colitis, ${ }^{26-28}$ we therefore examined whether pDCs would accumulate in colonic LP after DSS treatment. Because the constitutive expression of BST2 was observed on the cell surface of certain
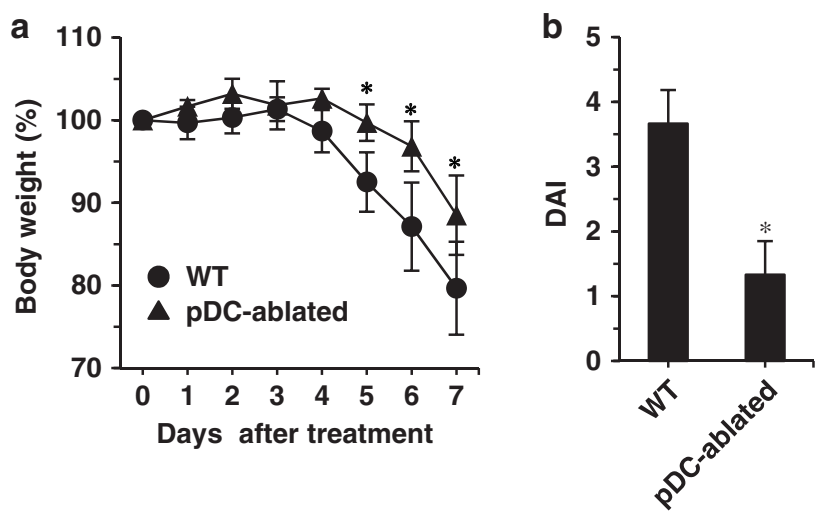

C

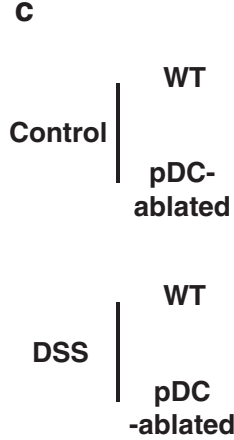

d

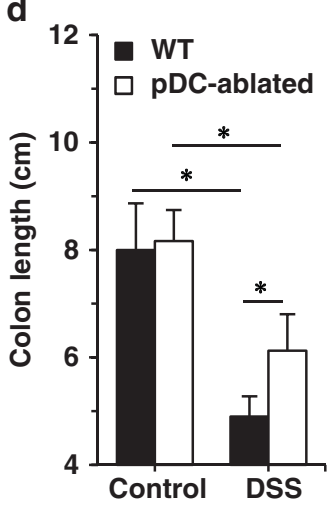

e

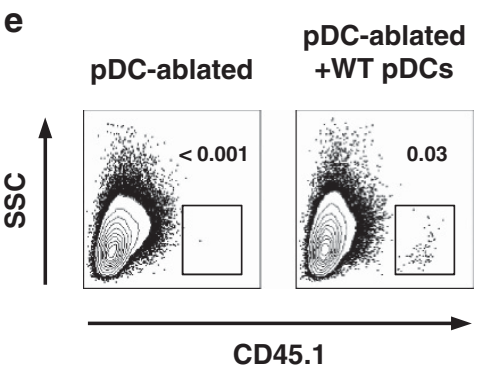

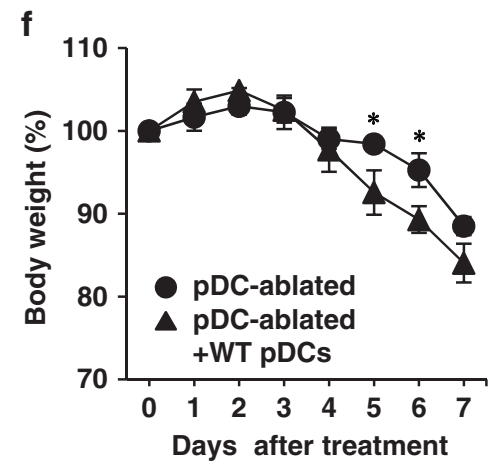

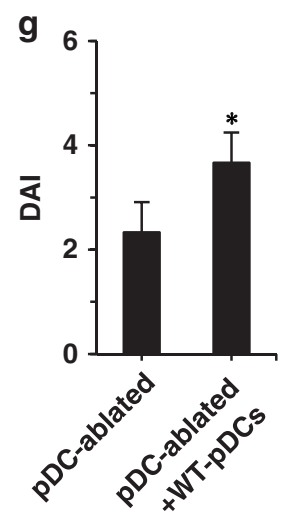

h

pDC-ablated

pDC-ablated +WT pDCs

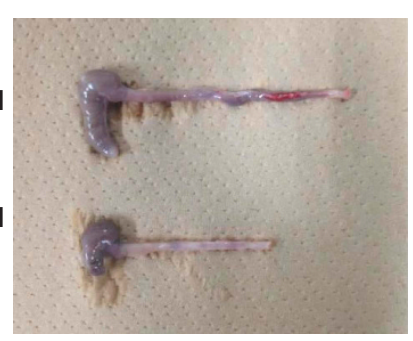


leukocytes other than pDCs in colonic LP (Figure 1c) in WT mice, intestinal pDCs were further detected as $\mathrm{B}_{220}{ }^{+} \mathrm{CD} 11 \mathrm{~b}^{-} \mathrm{CD} 11 \mathrm{c}^{\text {int }} \mathrm{BST} 2{ }^{+}$cells (R1: Supplementary Figure S3) to discriminate pDCs from other leukocytes in colonic LP (Figure 4a). Although pDCs barely existed in colonic LP under steady-state conditions, DSS treatment triggered the accumulation of pDCs in these intestinal mucosal tissues in WT mice, whereas the number of pDCs in the MLN was decreased inversely (Figure $\mathbf{4 b}$ and Supplementary Figure S3). Similarly, oral administration of DSS also induced the mobilization of I-A/I-E (MHC class II) ${ }^{+} \mathrm{CD} 11 \mathrm{c}^{\text {high }} \mathrm{CD} 64^{-}$cDCs (R2: Supplementary Figure S3), I-A/I-E ${ }^{+} \mathrm{CD} 11 \mathrm{c}^{+} \mathrm{CD} 64^{+}$macrophages (R3: Supplementary Figure S3), and I-A/I-E $\mathrm{E}^{-} \mathrm{CD} 11 \mathrm{~b}^{+}$Ly6C $\mathrm{C}^{\text {high }}$ inflammatory monocytes (R4: Supplementary Figure S3) into the inflamed colonic LP and MLN (Figure 4c-e). We confirmed that Siglec-H was predominantly expressed on $\mathrm{B}_{22}{ }^{+} \mathrm{CD}_{11 b^{-} \mathrm{CD} 11 \mathrm{c}^{\text {int }} \mathrm{BST}^{+}}{ }^{+} \mathrm{pDCs}$ in $\mathrm{LP}$ and MLN in WT mice during DSS treatment, whereas pDC-ablated mice exhibited specific elimination of $\mathrm{B}_{220}{ }^{+} \mathrm{CD}_{11} \mathrm{~b}^{-} \mathrm{CD} 11 \mathrm{c}^{\text {int }} \mathrm{BST} 22^{+}$pDCs, but not other leukocytes, in these intestinal mucosal tissues under DSS-induced inflamed conditions (Supplementary Figures S3, S4, and S5).

We further compared the cell surface expression of various molecules on pDCs in Spl, MLN, and colonic LP in WT mice before and after DSS treatment. In the steady-state conditions, pDCs in Spl and MLNs expressed Siglec-H, CD62L, CXCR3, and $\mathrm{C}-\mathrm{C}$ chemokine receptor 2 (CCR2) as well as CCR9 and $\alpha 4 \beta 7$, which are known as gut-homing receptors ${ }^{35}$ (Figure $4 \mathrm{f}$ and Supplementary Figure S3). Oral administration of DSS reduced the expression of CXCR3 and CD62L on MLN pDCs, whereas the expression of other molecules did not change (Figure 4f and Supplementary Figure S3). Furthermore, the accumulated colonic LP pDCs expressed Siglec-H, CD62L, CD69 known as activation inducer molecule, ${ }^{36}$ CCR2, CCR9, and $\alpha 4 \beta 7$ (Figure 4 f and Supplementary Figure S3). On the other hand, similar cell surface expression levels of I-A/I-E and costimulatory molecules were observed on pDCs in MLN and colonic LP in WT mice under both DSS-induced inflamed and noninflamed conditions (Supplementary Figures S3 and S6).

To clarify how pDCs mobilize into inflamed colonic LP, we examined the transcriptional expressions of chemokines in colonic explants from untreated and DSS-treated WT mice (Figure 5). The transcriptonal expression levels of $C c l 2$ ( Mcp1),
Ccl3 (Mip1a), Ccl5 (Rantes), Ccl7 (Mcp3), Ccl8 (Mcp2), Ccl25 (Teck), Cxcl9 (Mig), Cxcl10 (Ip10) and Cxcl11 (Itac) were enhanced in colonic explants during the development of DSSinduced acute colitis in WT mice.

Taken together, these results indicate that pDCs accumulate in the inflamed colonic LP possibly mediated through chemokine-dependent trafficking.

\section{pDCs mobilize into the DSS induce inflamed LP independent of lymphatic trafficking}

It has been shown that pDCs can accumulate in the peripheral LN tissues with their transit time, which is regulated by lysophospholipid sphingoshine-1-phosphate (S1P) and its receptors (S1PRs), both under homeostatic conditions and during viral infection, associating with a reduction of circulating pDCs in the blood. ${ }^{36}$ To further address whether pDCs utilize S1PR-mediated signaling for entry into the inflamed colon from peripheral and mucosal other LNs, we examined the influence of FTY720, an agonist for S1PRs to inhibit leukocyte egress from LNs, ${ }^{36}$ on the accumulation of pDCs in colonic LP in DSS-treated WT mice. The accumulation of $\mathrm{B}_{22}{ }^{+} \mathrm{CD}_{11 \mathrm{~b}}{ }^{-} \mathrm{CD} 11 \mathrm{c}^{\mathrm{int}} \mathrm{BST} 2{ }^{+}$pDCs in colonic LP was not influenced by FTY720 treatment under steady-state and inflammatory conditions caused by oral administration of DSS (Figure 6a,b and Supplementary Figure S3), in a manner similar to that observed for I-A/I$\mathrm{E}^{+} \mathrm{CD} 11 \mathrm{c}^{\text {high }} \mathrm{CD} 64^{-} \mathrm{cDCs}$ and I-A/I-E ${ }^{-} \mathrm{CD} 11 \mathrm{~b}^{+}$Ly6C $^{\text {high }}$ inflammatory monocytes (Figure 6c,d and Supplementary Figure S3). After oral DSS challenge, $\mathrm{CD} 3 \varepsilon^{+} \mathrm{T}$ cell also accumulated in colonic LP, which was suppressed by FTY720 treatment (Figure 6e and Supplementary Figure S3). On the other hand, FTY720 treatment inhibited the homeostatic retention of $\mathrm{B} 220^{+} \mathrm{CD} 11 \mathrm{~b}^{-} \mathrm{CD} 11 \mathrm{c}^{\mathrm{int}} \mathrm{BST} 2^{+}$pDCs within MLN, whereas DSS treatment resulted in the decreased number of $\mathrm{B}_{2} 20^{+} \mathrm{CD} 11 \mathrm{~b}^{-} \mathrm{CD} 11 \mathrm{c}^{\text {int }} \mathrm{BST}^{+}{ }^{+} \mathrm{pDCs}$ in MLN (Figure 6a,f and Supplementary Figure S3). We also observed that oral application of DSS enhanced the retention of $\mathrm{I}-\mathrm{A} / \mathrm{I}-\mathrm{E}^{+} \mathrm{CD} 11 \mathrm{c}^{\text {high }} \mathrm{CD} 64^{-} \quad \mathrm{cDCs}$ and I-A/I-E ${ }^{-} \mathrm{CD} 11 \mathrm{~b}^{+}{ }^{-} \mathrm{Ly} 6 \mathrm{C}^{\text {high }}$ inflammatory monocytes within MLN, whereas FTY720 treatment had little or no effect on their retention under physiological or inflammatory conditions (Figure 6g,h and Supplementary Figure S3). However, neither FTY720 nor DSS treatment had a minimal effect on the number of $\mathrm{CD} 3 \varepsilon^{+} \mathrm{T}$ cells in MLN (Figure 6i and Supplementary Figure S3).

\footnotetext{
Figure 2 pDCs aggravate DSS-induced acute colitis. (a-d) WT $(n=4)$ and pDC-ablated mice $(n=4)$ that had been treated with DT on 1 day before DSS treatment and day 3 after the start of the treatment were orally administrated with $2 \%$ DSS for 7 days, and the disease progression was monitored. Changes in BW (a), DAI (b), the picture of colon (c), and length of colon (d) were plotted. (e-h) pDC-ablated mice $(n=4)$ that had been treated with DT and adoptively transferred with or without CD $45.1^{+}$WT pDCs as prepared above on 1 day before DSS treatment and day 3 after the start of the treatment were orally administrated with $2 \%$ DSS for 7 days. (e) The frequency of CD $45.1^{+}$cells in the colonic LP was analyzed by flow cytometry on day 7 after the start of DSS treatment. Data are presented as a dot plot, and numbers represent the proportion of the indicated cell populations among B220 ${ }^{+} \mathrm{CD}_{11 \mathrm{~b}}{ }^{-} \mathrm{Cells}^{\mathrm{in}}$ each quadrant. (f-h) Changes in BW (f), DAI (g), and the picture of colon $(\mathbf{h})$ were plotted. Data are the mean \pm s.d. from three individual samples in a single experiment. ${ }^{*} P<0.01$ compared with WT mice. All data are representative at least three independent experiments. BW, body weight; DAl, disease activity index; DT, diphtheria toxin; DSS, dextran sodium sulfate; LP, lamina propria; pDC, plasmacytoid dendritic cell; WT, wild type.
} 
Taken together, these results indicate that pDCs mobilize into the inflamed colonic LP independent of S1PR-mediated signaling.

\section{pDCs promote the mobilization of inflammatory phagocytes into the inflamed colon LP}

Given the contribution of pDCs to intestinal inflammation for the development of acute colitis, we asked whether pDCs would have an influence on the recruitment of inflammatory phagocytes on day 7 after the start of DSS treatment. Although DSS treatment induced the recruitment of CD11 ${ }^{+}$cells to the inflamed colonic LP in WT mice, pDC-ablated mice exhibited the reduction of this infiltration of myeloid cells (Figure 7a and Supplementary Figure S3). Immunohistochemical analysis also demonstrated that $\mathrm{CD} 11 \mathrm{~b}^{+}$cells infiltrated into both LP and intraepithelial region in the inflamed colon of WT mice, whereas their accumulations were diminished in pDC-ablated mice (Figure 7b).

We further assessed the role of pDCs in the accumulation of cDCs, macrophages and inflammatory monocytes as well as other leukocytes in the inflamed colon LP in DSS-induced acute colitis. DSS treatment triggered the recruitment of I-A/I-E ${ }^{-} \mathrm{CD} 11 \mathrm{~b}^{+} \mathrm{Ly}_{6 \mathrm{G}}{ }^{+}$neutrophils (R5: Supplementary Figure S3) and $\mathrm{I}-\mathrm{A} / \mathrm{I}-\mathrm{E}^{-} \mathrm{CD} 11 \mathrm{~b}^{+}$Siglec- $\mathrm{F}^{+}$eosinophils (R6: Supplementary Figure S3) in addition to I-A/I$\mathrm{E}^{+} \mathrm{CD} 11 \mathrm{c}^{\text {high }} \mathrm{CD} 64^{-} \quad \mathrm{cDCs}, \quad \mathrm{I}-\mathrm{A} / \mathrm{I}-\mathrm{E}^{+} \mathrm{CD} 11 \mathrm{c}^{+} \mathrm{CD} 64^{+}$ macrophages, and I-A/I-E ${ }^{-} \mathrm{CD}_{11} \mathrm{~b}^{+}$Ly6 $^{\text {high }}$ inflammatory monocytes as well as lymphocytes, including $\mathrm{CD} 3 \varepsilon^{+} \mathrm{T}$ cells and $\mathrm{CD} 19^{+} \mathrm{B}_{22} 0^{+} \mathrm{B}$ cells in WT mice (Figure 7c and Supplementary Figure S3). In contrast, these mobilizations of I-A/I-E ${ }^{+} \mathrm{CD} 11 \mathrm{c}^{\mathrm{high}} \mathrm{CD} 64^{-}$cDCs, I-A/I-E ${ }^{+} \mathrm{CD} 11 \mathrm{c}^{+} \mathrm{CD} 64^{+}$ macrophages, and I-A/I-E ${ }^{-} \mathrm{CD} 11 \mathrm{~b}^{+} \mathrm{Ly}_{6} \mathrm{C}^{\text {high }}$ inflammatory monocytes into the inflamed colonic LP were attenuated in pDC-ablated mice when compared with WT mice, whereas the infiltrations of $\mathrm{I}-\mathrm{A} / \mathrm{I}-\mathrm{E}^{-} \mathrm{CD} 11 \mathrm{~b}^{+} \mathrm{Ly}_{6 \mathrm{G}}{ }^{+}$neutrophils and

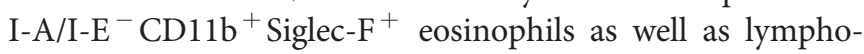
cytes were comparable between WT and pDC-ablated mice (Figure 7c and Supplementary Figure S3). We also observed similar frequency of $\mathrm{CD}^{+}{ }^{+}$effector $\mathrm{T}\left(\mathrm{T}_{\text {eff }}\right)$ cells, including IFN- $\gamma$-producing $\mathrm{CD} 4^{+} \mathrm{T}$ cells (T-helper $1 ; \mathrm{T}_{\mathrm{H}} 1$ cells) and IL-17-producing $\mathrm{CD}^{+} \mathrm{T}$ cells $\left(\mathrm{T}_{\mathrm{H}} 17\right.$ cells), as well as $\mathrm{CD}^{+}{ }^{+} \mathrm{Foxp}^{+}$regulatory $\mathrm{T}\left(\mathrm{T}_{\mathrm{reg}}\right)$ cells in the inflamed colon between WT and pDC-ablated mice (Supplementary Figure S7).

Collectively, these results indicate that the deficiency of pDCs inhibits the recruitment of inflammatory phagocytes to the inflamed colon.

The development of acute colitis reportedly requires the mobilization and accumulation of macrophage and inflammatory monocytes into the inflamed colon through the local production of CCR2 ligands by colonic ECs (cECs), such as CCL2, CCL7, and CCL8. ${ }^{27,28}$ We therefore compared the colonic production of CCR2 ligands between $\mathrm{WT}$ and pDCablated mice following DSS treatment. pDC-ablated mice exhibited the marked reduction of the transcriptional expressions of $\mathrm{Ccl} 2$, but not $\mathrm{Ccl} 7$ and $\mathrm{Ccl}$, in colon explants after oral administration of DSS than WT mice (Figure 7d). Indeed, the production of CCL2 from colonic explants was much lower in pDC-ablated mice than WT mice following DSS treatment (Figure 7e).

Collectively, these results indicate that the deficiency of pDCs reduces the production of CCL2 in the inflamed colon.

\section{DISCUSSION}

Although the contributions of intestinal monocytes/macrophages and cDCs in the inflamed colon have been appreciated for the development of T-cell-independent acute colitis, ${ }^{26-28}$ the mechanism responsible for the control of colonic inflammation and the function of distinct subsets of colitogenic phagocytes is still elusive. In this study, we revealed a critical function for pDCs in the induction of the mucosal inflammation that regulate the colonic accumulation of inflammatory phagocytes, leading to the initiation and aggravation of acute colitis.

The contributions of certain subpopulations of DC and macrophage lineages to the regulation of the development of acute colitis have been elucidated by utilizing Tg mouse lines endowed with the expression of DTR under the control of DCor macrophage-related genes, such as $\operatorname{Itgax}{ }^{26} \mathrm{Cd} 169,{ }^{28,37}$ Clec $4 a 4,{ }^{37}$ and $\operatorname{Clec} 9 a,{ }^{37}$ whereas the phenotype of Batf 3 deficiency did not show any exacerbated inflammation during acute colitis. ${ }^{38}$ Series of our studies have shown that pDCablated mice exhibited a near-complete and specific elimination of pDCs in the lymphoid tissues following DT treatment, but they retained other leukocytes, including $\mathrm{CDC}$ subsets. ${ }^{31,32}$ Analysis of pDC-ablated mice revealed that the development of DSS-induced acute colitis was attenuated in the absence of pDCs. Although we would not absolutely rule out the possibility that some minor unidentified populations of Siglec $-\mathrm{H}^{+}$hematopoietic cells affecting extent and severity of acute colitis might be ablated in pDC-ablated mice, the amelioration of clinical symptom of acute colitis in pDCablated mice was overcome by reconstitution with pDCs. Thus, these findings strongly suggest that pDCs as well as CDCs are prerequisite for the initiation and aggravation of DSS-induced acute colitis. However, the milder development of DSS-induced acute colitis was occurred even in the absence of pDCs or cDCs, implying that other cell types, such as macrophages, inflammatory monocytes, neutrophils, and eosinophils are also participated in the development of T-cell-independent acute colitis as these leukocytes accumulated into the inflamed colonic LP.

Disruption of epithelial barrier and subsequent translocation of luminal microbiota into LP have been suggested to provoke intestinal inflammation mediated by the production of cytokines from host myeloid cells. ${ }^{26-28}$ Notably, the deficiency of pDCs abrogates colonic inflammation and pathological symptom of acute colitis as well as infiltration of inflammatory leukocytes into inflamed colon in DSS-fed mice. We have previously shown that $\mathrm{pDCs}$ could be the primary inflammatory cells to produce cytokines upon recognition of appropriate endosomal TLR ligands and viral components for the induction 
a
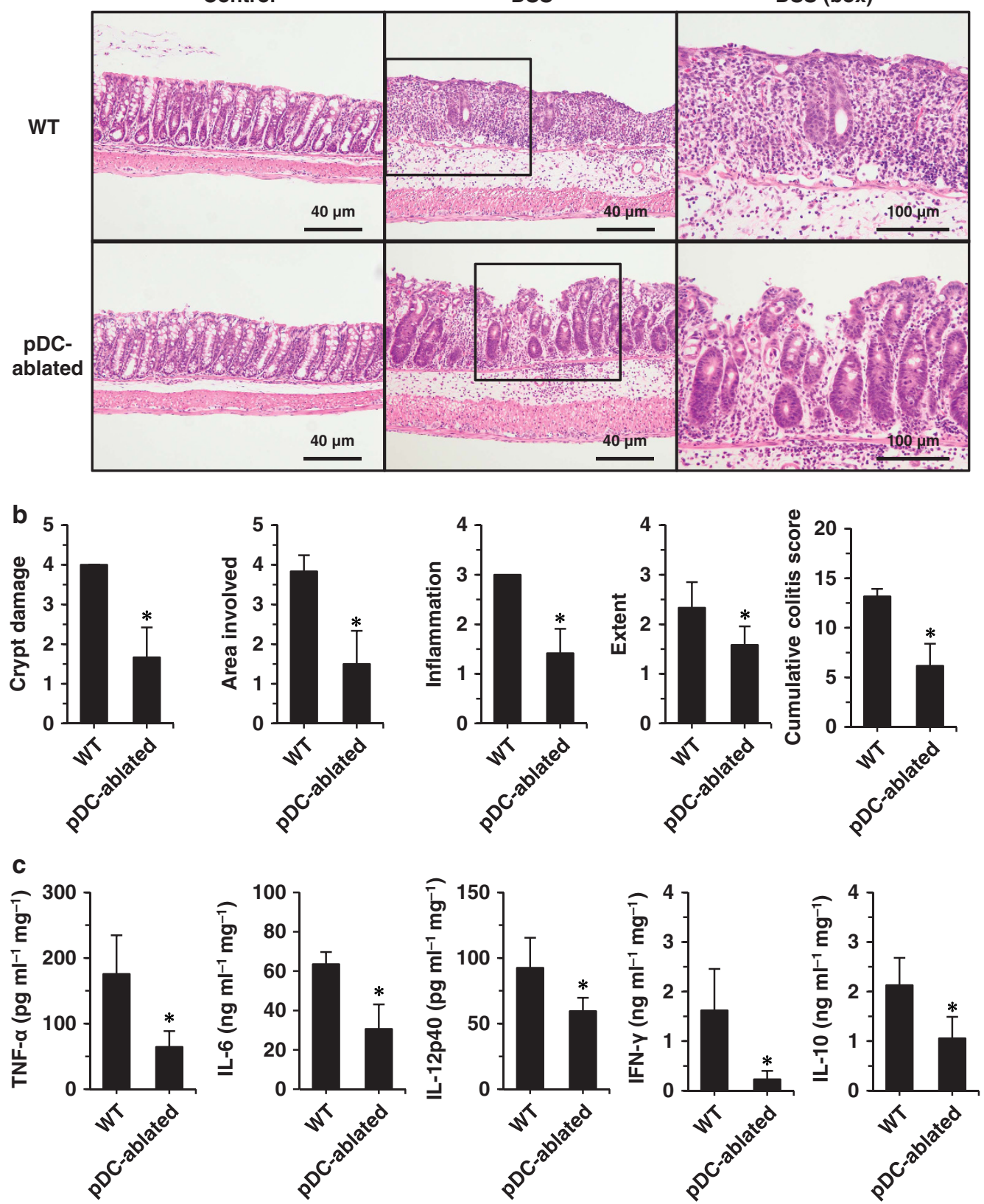

Figure 3 pDCs promote DSS-induced colonic inflammation. WT $(n=4)$ and pDC-ablated mice $(n=4)$ that had been treated with DT on 1 day before DSS treatment and day 3 after the start of the treatment were treated with or without $2 \%$ DSS for 7 days. (a) H\&E staining of the paraffin-embedded sections of middle colon obtained from WT (upper panel) and pDC-ablated mice (lower panel) with low-magnification $(\times 4)$ and further magnification ( $\times 10)$. (b) Histological scoring for crypt damage (0-4), area involved (1-4), inflammation (0-3), extent (0-3), and cumulative score of middle colon obtained from WT and pDC-ablated mice are plotted. Data are the mean \pm s.d. from three individual samples in a single experiment. ${ }^{\star} P<0.01 \mathrm{compared}$ with WT mice. (c) The production of cytokines in the cultured middle colonic explants obtained from DSS-fed WT and pDC-ablated mice was measured by ELISA. Data are the mean \pm s.d. from three individual samples in a single experiment. ${ }^{*} P<0.01$ compared with WT mice. All data are representative at least three independent experiments. DT, diphtheria toxin; DSS, dextran sodium sulfate; H\&E, hematoxylin and eosin; pDC, plasmacytoid dendritic cell; ELISA, enzyme-linked immunosorbent assay; WT, wild type.

of systemic inflammation. ${ }^{31,32}$ Collectively, these findings suggest that $\mathrm{pDCs}$ are required for initiation of DSS-induced colonic inflammation and tissue damage possibly mediated through the control of mucosal production of proinflammatory cytokines. On the other hand, Mizuno et al. ${ }^{39}$ have recently suggested that $\mathrm{CCR}^{+}$pDCs in the small intestine (SI) suppress the development of murine spontaneous intestinal inflammation as $\mathrm{CCR}^{+}{ }^{+}$pDCs preferentially resided in the SI in the steady state, and $\mathrm{C} C \mathrm{r} 9^{-1-} \mathrm{Rag}-2^{-1-}$ mice developed more severe ileitis compared with Rag- $2^{-1-}$ mice when these mice were adoptively transferred with $\mathrm{CD} 4{ }^{+} \mathrm{CD} 45 \mathrm{RB}^{\text {high }}$ $\mathrm{T}$ cells, although they did not assess the direct contribution 
a WT

pDC-ablated
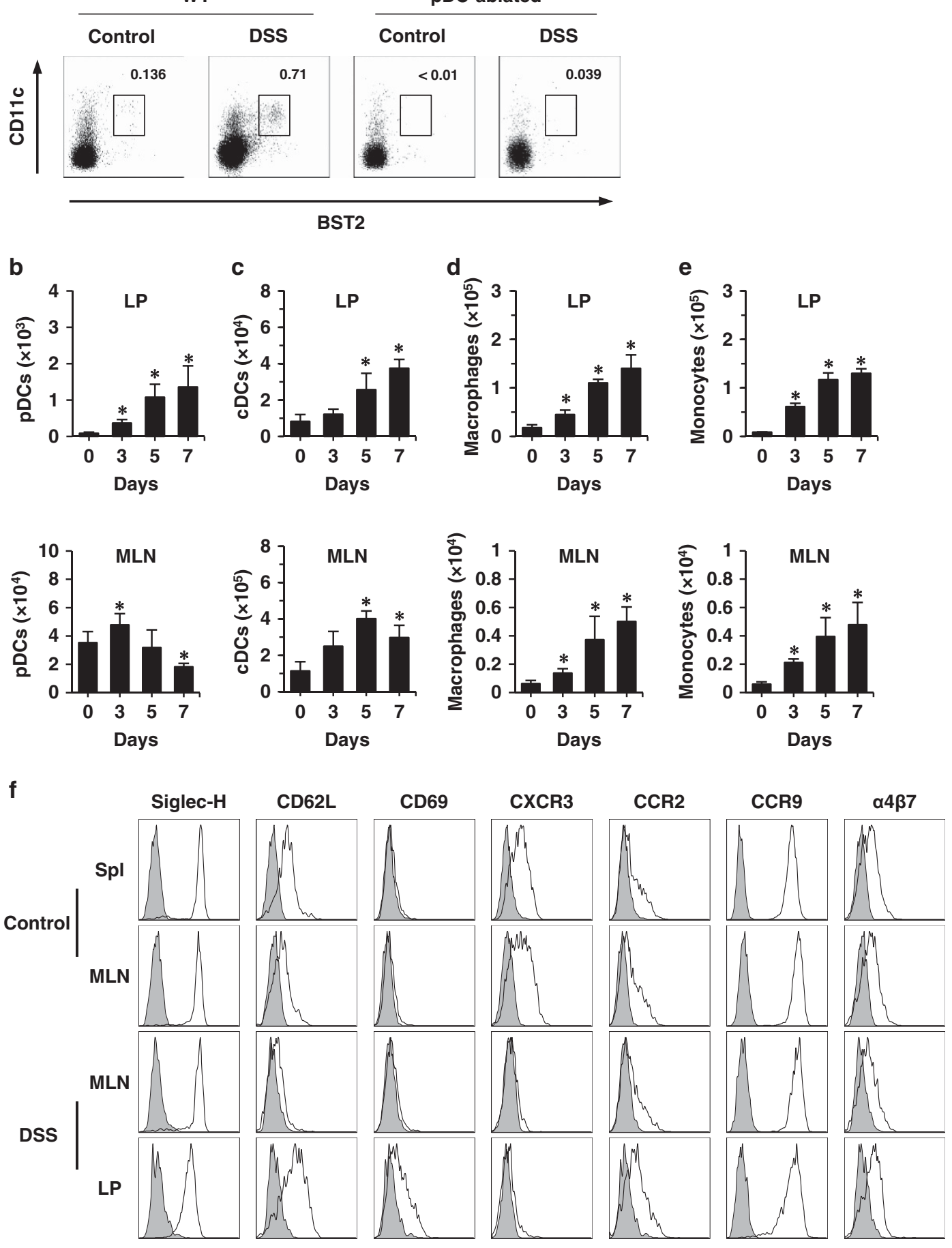

Figure 4 pDCs accumulate in the colon LP after oral administration of DSS. (a) WT $(n=4)$ and pDC-ablated mice $(n=4)$ that had been treated with DT on 1 day before DSS treatment and day 3 after the start of the treatment were orally administrated with or without $2 \%$ DSS for 7 days, and then the frequency of pDCs in colonic LP was analyzed by flow cytometry on day 7 after the start of DSS treatment. Data are represented as a dot plot, and numbers represent the proportion of $\mathrm{B} 220^{+} \mathrm{CD} 11 \mathrm{~b}^{-} \mathrm{CD} 11 \mathrm{c}^{\text {int }} \mathrm{BST} 2^{+} \mathrm{pDC}$ among CD45.2 ${ }^{+}$leukocytes. (b-e) WT mice $(n=4)$ that had been treated with DT on 1 day before DSS treatment and day 3 after the start of the treatment were orally administrated with or without $2 \%$ DSS for 7 days, and absolute cell numbers of $\mathrm{B} 220^{+} \mathrm{CD}_{11} \mathrm{~b}^{-} \mathrm{CD} 11 \mathrm{c}^{\text {int }} \mathrm{BST} 2^{+} \mathrm{pDCs}(\mathbf{b}), \mathrm{I}-\mathrm{A} / \mathrm{I}-\mathrm{E}^{+} \mathrm{CD} 11 \mathrm{c}^{\text {high }} \mathrm{CD} 64^{-} \mathrm{CDCs}(\mathbf{c}), \mathrm{I}-\mathrm{A} / \mathrm{I}-\mathrm{E}^{+} \mathrm{CD} 11 \mathrm{c}^{+} \mathrm{CD}^{-} 4^{+}$macrophages $(\mathbf{d})$, and $\mathrm{I}-\mathrm{A} / \mathrm{I}-\mathrm{E}^{-} \mathrm{CD} 11 \mathrm{~b}^{+} \mathrm{Ly} 6 \mathrm{C}^{\text {high }}$ inflammatory monocytes (e) in the colonic LP (upper panel) and MLN (lower panel) were analyzed by flow cytometry at indicated times after the start of DSS treatment. Data are the mean \pm s.d. from three individual samples in a single experiment. ${ }^{\star} P<0.01$ compared with untreated WT mice. (f) The expression of cell surface molecules on $\mathrm{B}_{22}{ }^{+} \mathrm{CD} 11 \mathrm{~b}{ }^{-} \mathrm{CD} 11 \mathrm{c}^{\text {int }} \mathrm{BST2}{ }^{+} \mathrm{pDC}$ in Spl, MLN, and colonic LP obtained from non-fed WT $(n=4)$ or DSS-fed WT mice $(n=4)$ on day 7 after the start of DSS treatment was analyzed by flow cytometry. Data are represented as a histogram. All data are representative at least three independent experiments. DT, diphtheria toxin; DSS, dextran sodium sulfate; LP, lamina propria; MLN, mesenteric lymph node; Spl, spleen; pDC, plasmacytoid dendritic cell; WT, wild type. 

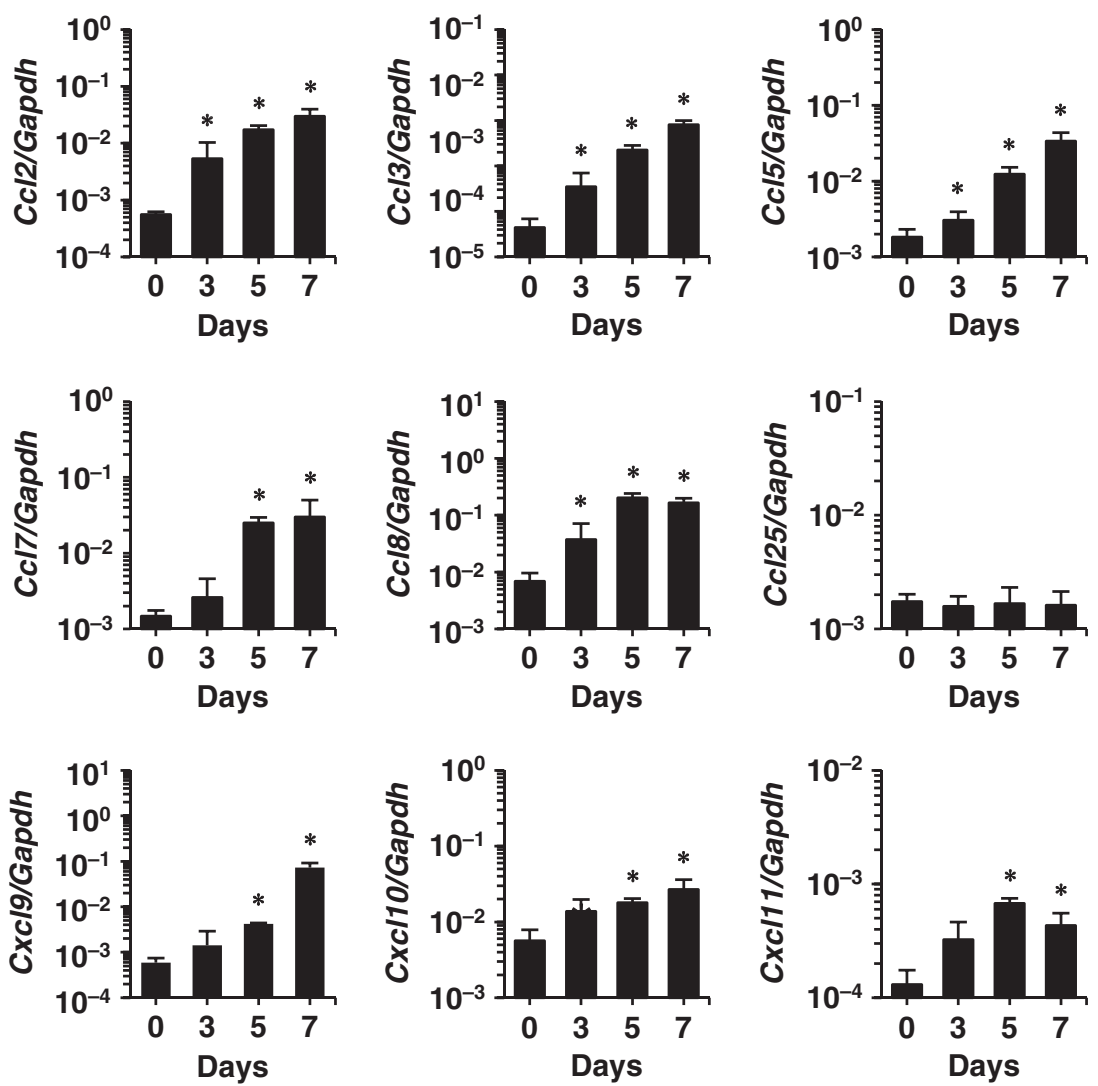

Figure 5 Oral administration of DSS promotes the colonic production of chemokines. WT mice $(n=4)$ that had been treated with DT on 1 day before DSS treatment and day 3 after the start of the treatment were orally administrated with or without $2 \%$ DSS, and transcriptional expression levels of chemokines in the middle colonic explants at indicated times after the start of DSS treatment were measured by quantitative reverse transcriptase (RT)PCR. Data are the mean \pm s.d. from three individual samples in a single experiment. ${ }^{*} P<0.01$ compared with untreated WT mice. All data are representative at least three independent experiments. DT, diphtheria toxin; DSS, dextran sodium sulfate; WT, wild type.

of $\mathrm{CCR}^{+}$pDCs to the regulation of the ileitis. This discrepancy regarding the role of pDCs in the control of the intestinal inflammation might be due to the different experimental designs (e.g., our T-cell-independent vs. their $\mathrm{T}$-cell-dependent murine models), although the precise reasons are still unclear.

Although IFN-I has been shown to mediate the regulatory role of pDCs in the control of several immune responses by affecting various functions of hematopoietic and non-hematopoietic cells, ${ }^{7-14}$ analysis of Ifnar ${ }^{-/-}$and Ifnar ${ }^{-/-}$pDCablated mice showed that the deficiency of Ifnarl in the presence or absence of pDCs had no apparent effect on the clinical symptom of DSS-induced acute colitis. Furthermore, the colonic production of IFN-I did not change under both DSS-induced inflamed and noninflamed conditions in the absence of pDCs, implying that stromal cells and ECs ${ }^{40}$ might be responsible for the enhanced production of IFN- $\beta$ in the DSS-induced inflamed colon. Similar to IFN-I treatment of IBD patients with mixed results, in which a few of clinical studies initially reported promising results but most of these studies failed to demonstrate beneficial therapeutic effect or showed even exacerbations, ${ }^{41}$ previous reports showed some controversies for the role of IFN-I signaling in the development of
DSS-induced colitis when Ifnar1 $1^{-/-}$mice were used for the comparison of WT mice. ${ }^{26,42,43}$ The reason why the IFN-I signaling has inconsistency for the control of DSS-induced acute colitis remains unclear, but the discrepancies might be explained by amount of oral application of DSS, ${ }^{43}$ experimental conditions, and environmental factors. Taken together, these results suggest that $\mathrm{pDCs}$ regulate the colonic inflammation independent of their pathophysiological production of IFN-I.

Despite extensive literature on the recruitment of the subpopulations of myeloid cells into the inflamed colon, ${ }^{26-28}$ the accumulation of pDCs in the inflamed mucosal tissues in the progression of acute colitis remains to be determined. Whereas pDCs rarely existed in colonic LP under homeostatic conditions, they mobilized into the inflamed colon upon DSS treatment, that was similar to that observed for CDCs as well as macrophages and inflammatory monocytes. Different from those of these inflammatory phagocytes, the proportion of pDCs was decreased in MLN in the progression of acute colitis, suggesting that pDCs could migrate from MLNs into other tissues and/or circulatory systems. Furthermore, mucosal pDCs expressed both several gut-homing receptors and inflammatory chemokine receptors, whereas their expression levels of MHC and costimulatory molecules did not change under steady-state 
a

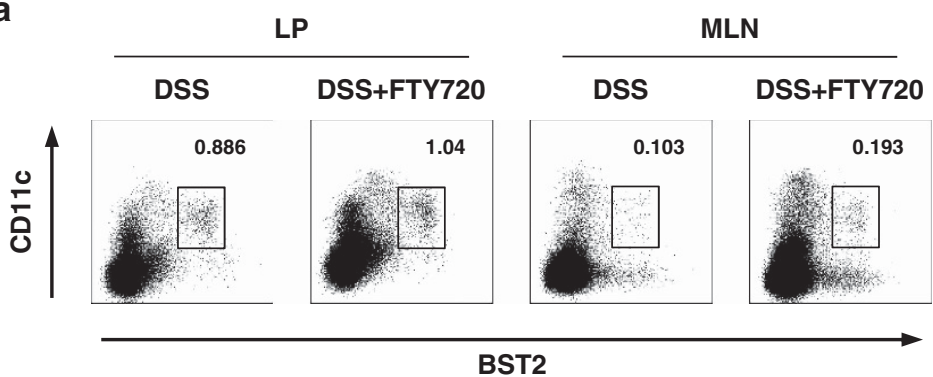

b

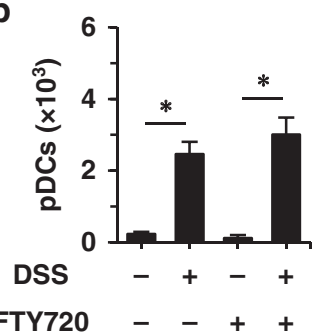

f

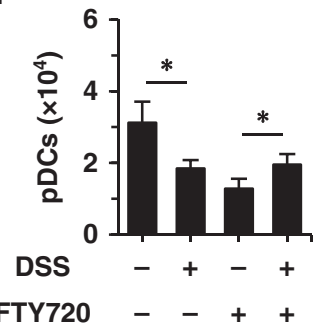

C

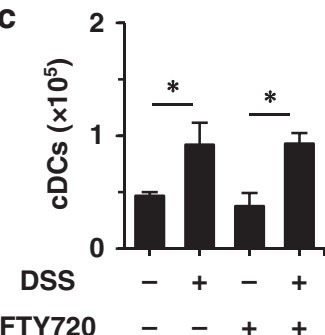

g

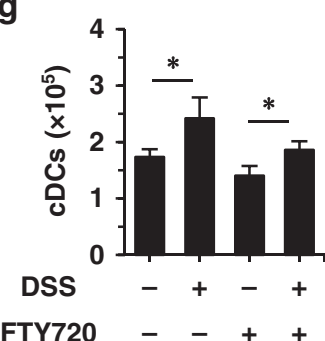

d

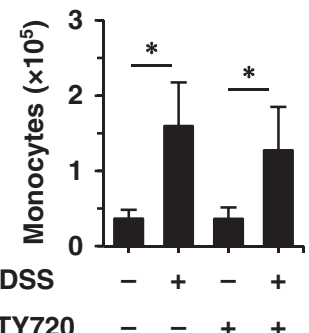

h

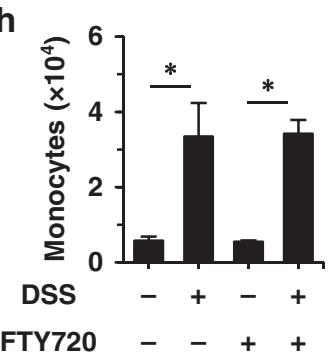

e

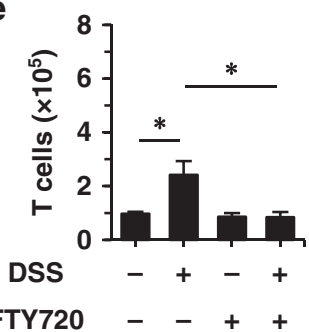

i

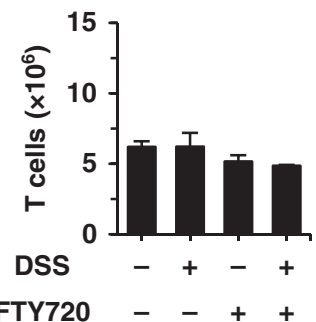

Figure 6 Influence of FTY720 treatment on the colonic accumulation of leukocytes. WT mice $(n=4)$ that had been treated with DT on 1 day before DSS treatment and day 3 after the start of the treatment were orally administrated with $2 \%$ DSS in combination with or without systemic injection of FTY720 for 7 days. (a) The frequency of CD11 $c^{\text {int }} \mathrm{BST} 2^{+}$pDCs in the MLN and B220 ${ }^{+} \mathrm{CD} 11 \mathrm{~b}^{-} \mathrm{CD} 11 \mathrm{c}^{\text {int }} \mathrm{BST} 2^{+}$pDCs in the colonic LP were analyzed by flow cytometry on day 7 after the start of DSS treatment. Data are represented as a dot plot, and numbers represent the proportion of the indicated cell population in each quadrant. $(\mathbf{b}-\mathbf{g})$ Absolute cell numbers of $\mathrm{B}_{2} 20^{+} \mathrm{CD} 11 \mathrm{~b}^{-} \mathrm{CD} 11 \mathrm{c}^{\text {int }} \mathrm{BST} 2^{+} \mathrm{pDCs}(\mathbf{b}, \mathbf{f}), \mathrm{I}-\mathrm{A} / \mathrm{I}-\mathrm{E}^{+} \mathrm{CD}^{+} 11 \mathrm{c}^{\text {high }} \mathrm{CD} 64^{-} \mathrm{cDCs}(\mathbf{c}, \mathbf{g})$, I-A/I-E ${ }^{-}$CD11 b ${ }^{+}$Ly6C $C^{\text {high }}$ inflammatory monocytes (d,h), and CD3 $\varepsilon^{+}$T cells $(\mathbf{e}, \mathbf{i})$ in the colonic LP (b-e) and MLN (f-i) were analyzed by flow cytometry on day 7 after the start of DSS treatment. Data are the mean \pm s.d. from three individual samples in a single experiment. ${ }^{\star} P<0.01$ compared with untreated WT mice. All data are representative at least three independent experiments. DT, diphtheria toxin; DSS, dextran sodium sulfate; MLN, mesenteric lymph node; LP, lamina propria; WT, wild type.

and inflammatory conditions triggered by DSS treatment. On the other hand, oral application of DSS upregulated the production of multiple inflammatory chemokines in the inflamed colon. Therefore, these results suggest that $\mathrm{pDCs}$ accumulate into the inflamed colonic LP by utilizing the integration of the gut-homing receptors and inflammatory chemokine receptors without altering their ability to generate $\mathrm{CD} 4{ }^{+} \mathrm{T}_{\text {eff }}$ cells and $\mathrm{CD} 4{ }^{+}$Foxp $3^{+} \mathrm{T}_{\text {reg }}$ cells during the development of acute colitis.

Accumulation of pDCs in the secondary LN tissues and their egress into lymphatic vessels has been reportedly regulated by S1P-dependent mechanism for migration as they selectively S1P4 among S1PRs. ${ }^{36}$ However, treatment with FTY720 failed to control the accumulation of pDCs as well as cDCs and inflammatory monocytes in colonic LP under homeostatic and inflammatory conditions caused by the administration of DSS. In contrast, systemic administration of FTY720 or oral application of DSS suppressed the accumulation of pDCs in MLN under steady-state or inflammatory conditions, which were different from the influence of FTY720 or DSS on the retention of cDCs and inflammatory monocytes within MLN. On the other hand, FTY720 treatment abrogated the DSSinduced enhanced accumulation of $\mathrm{T}$ cells in the inflamed colonic LP, whereas it had no effect their retention in MLNs, implying that T cells might recruit into the inflamed colonic LP from other secondary LN tissues than MLN. Thus, these findings suggest that $\mathrm{pDCs}$ as well as $\mathrm{CDCs}$ and inflammatory monocytes accumulate into the inflamed colonic LP through hematogenous mobilization rather than S1P4-dependent lymphatic trafficking, whereas pDCs mobilized into MLN through blood and lymphatic vessels under homeostatic and mucosal inflammatory conditions.

Among various types of hematopoietic cells infiltrating into inflammatory mucosal tissues, the accumulation of monocytes/ macrophages and cDCs in the inflamed colon has been considered as key phase to induce T-cell-independent acute colitis mediated through the intestinal production of CCR2 ligands. ${ }^{27,28}$ However, how the mobilization of these 
a

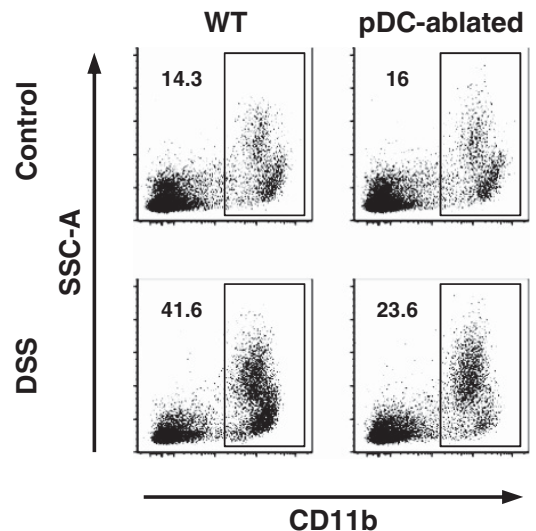

C
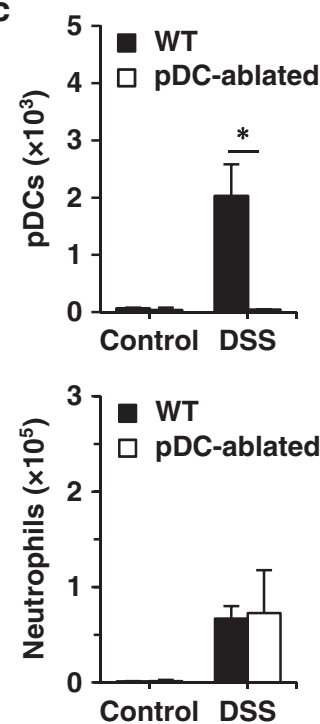

d

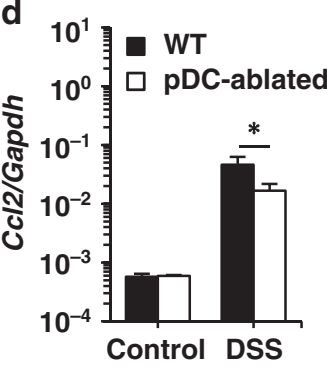

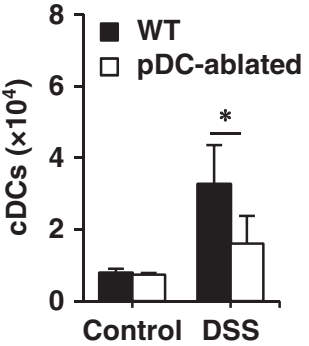
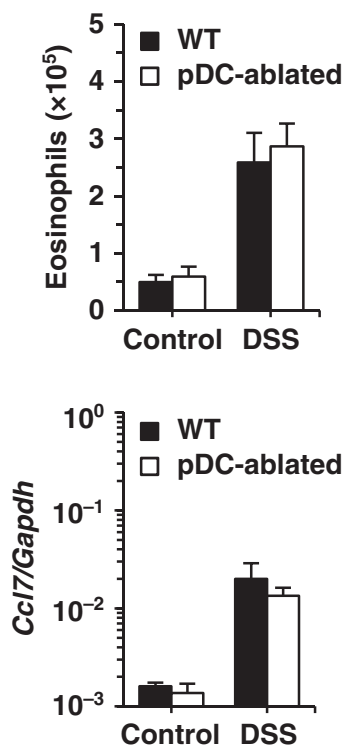

b

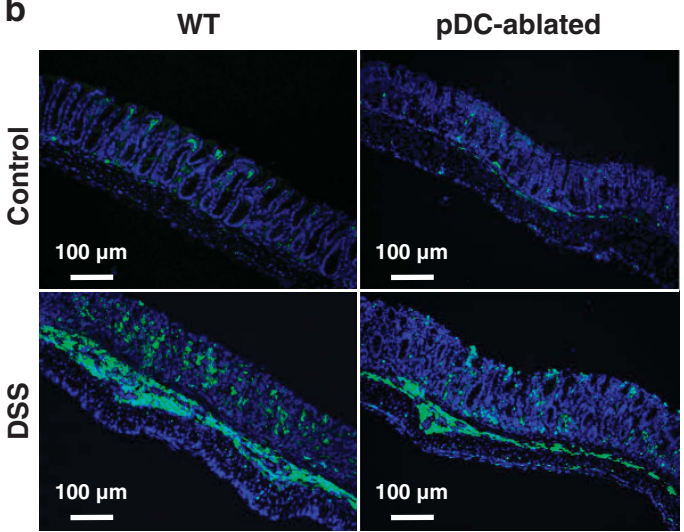

CD11b/DAPI
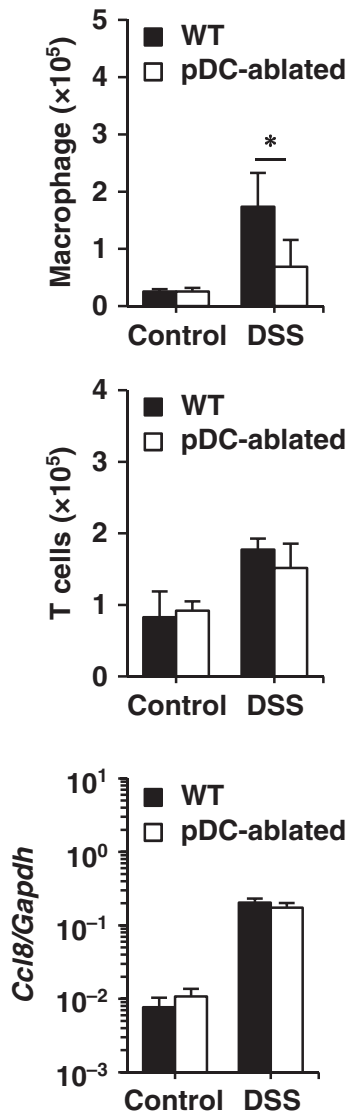
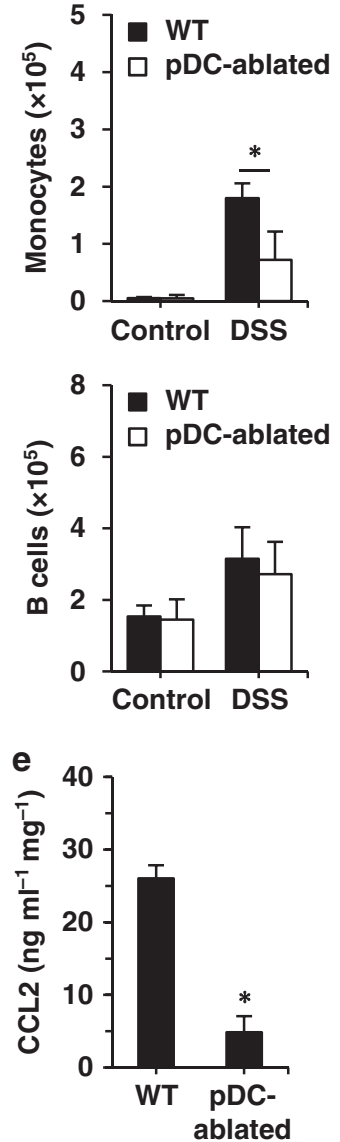

Figure 7 pDCs induce the recruitment of inflammatory monocytes and macrophages into the inflamed colonic LP. WT $(n=4)$ and pDC-ablated mice $(n=4)$ that had been treated with DT on 1 day before DSS treatment and day 3 after the start of the treatment were orally administrated with or without $2 \%$ DSS for 5 days. (a) The frequency of CD11 ${ }^{+}$cells in colonic LP obtained from untreated (upper panel) or DSS-treated (lower panel) mice were analyzed by flow cytometry on day 5 after the start of DSS treatment. Data are represented as a dot plot, and numbers represent the proportion of CD11 $\mathrm{b}^{+}$cells among CD45.2 ${ }^{+}$ leukocytes in each quadrant. (b) Immunofluorescent microscopic analysis was performed on frozen horizontal sections (magnification; $\times 10)$ of middle colon obtained from untreated (upper panel) or DSS-treated (lower panel) mice on day 5 after the start of DSS treatment. Sections were stained for DAPI

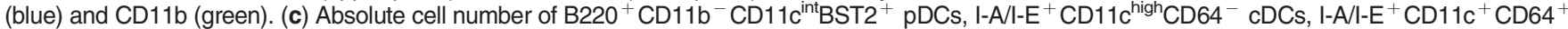

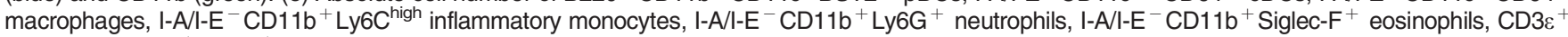
T cells, and B220 ${ }^{+} \mathrm{CD} 19^{+} \mathrm{B}$ cells in the colonic LP obtained from WT and pDC-ablated mice were analyzed by flow cytometry on day 5 after the start of DSS treatment. Data are the mean \pm s.d. from three individual samples in a single experiment. ${ }^{*} P<0.01$ compared with WT mice. (d) Transcriptional expression levels of CCR2 ligands in the middle colonic explants obtained from WT and pDC-ablated mice were measured by quantitative RT-PCR. Data are the mean \pm s.d. from three individual samples in a single experiment. ${ }^{*} P<0.01$ compared with WT mice. (e) The production of CCL2 in the cultured middle colonic explants obtained from DSS-fed WT mice and pDC-ablated mice was measured by ELISA. Data are the mean \pm s.d. from three individual samples in a single experiment. ${ }^{*} P<0.01$ compared with WT mice. All data are representative at least three independent experiments. DAPI, 4,6-diamidino-2-phenylindole; DSS, dextran sodium sulfate; pDC, plasmacytoid dendritic cell; LP, lamina propria; RT-PCR; reverse transcription-PCR; WT, wild type. 
inflammatory phagocytes is regulated remains to be determined. Importantly, the deficiency of pDCs markedly impaired the recruitment of inflammatory monocytes/macrophages and cDCs, but not other leukocytes, into the inflamed colonic LP after DSS treatment. Furthermore, the cEC-derived mucosal production of CCL2, but not other CCR2 ligands, was specifically diminished in the absence of pDCs under DSSinduced inflammatory conditions. Collectively, these results suggest that $\mathrm{pDCs}$ act as upstream regulators to recruit some populations of $\mathrm{CCR} 2^{+}$inflammatory phagocytes to the inflamed colon possibly mediated by the control of cEC-derived mucosal production of CCL2. On the other hand, the intestinal inflammation and accumulation of the phagocytes were still observed under the deficiency of pDCs or CCR2. ${ }^{27}$ These phenomena imply that pDC- and/or CCL2independent axis might also control the colonic mobilization of inflammatory phagocytes for the development of intestinal inflammation.

In conclusion, we describe that pDCs have a crucial role in the initiation and exacerbation of T-cell-independent acute colitis mediated by promoting intestinal inflammation. Our findings suggest that pDCs function as the primary and central cells to regulate the mobilization of colitogenic phagocytes into the inflamed intestinal tissues for the progression of colonic inflammation upon sensing epithelial injury triggered by genetic and environmental factors during the development of acute colitis, and therefore they are instrumental in regulating gut mucosal immune homeostasis. Thus, pDCs represent an attractive valid cellular target for the intervention and treatment of IBD to improve outcome for these patients.

\section{METHODS}

Mice. The following 8-12-week-old female mice were used in this study: C57BL/6 (Clea Japan, Tokyo, Japan), B6.CD45.1 (Charles River Laboratories, Portage, MI), B6.FVB-Tg ${ }^{\text {Itgax }-D T R / E G F P ~} \mathrm{Lan} / \mathrm{J}$ (CD11cDTR/EGFP) ${ }^{33}$ (referred to as CDC-ablated mice), and B6.Ifnar $1^{-1-}$ mice. $^{34}$ B6.Cg-Siglech ${ }^{<\mathrm{tm} 1.1 \mathrm{Ksat}>}$ mice (B6.Siglec-H-DTR mice) generated as described previously ${ }^{31}$ were deposited in RIKEN BioResource Center (accession number; RBRC05658), and their littermates were used as WT mice. For the systemic ablation of cDCs or pDCs, B6.CD11c-DTR/EGFP mice or B6.Siglec-H-DTR mice were intraperitoneally (i.p.) injected with DT (100 ng per mouse or $1 \mu \mathrm{g}$ per mouse, respectively; Sigma-Aldrich, St. Louis, MO) on 1 day before DSS treatment and day 3 after the start of the treatment. B6.WT mice were also treated with DT as controls. In some experiments, B6.Siglec-H-DTR mice were i.p. injected with DT (1 $\mu$ g per mouse) on day 4 after initiation of DSS application. B6. Ifnar $1^{-/}-$Siglec-H-DTR mice were bred in-house by crossing B6.Siglec-H-DTR mice with B6.Ifnar ${ }^{-1-}$ mice. All mice were bred and maintained in specific pathogen-free conditions in the animal facility at University of Miyazaki, and all experiments were performed in accordance with institutional guidelines of the Animal Experiment Committee and Gene Recombination Experiment Committee.

Cell isolation and culture. To prepare single-cell suspensions, Spl and MLN were digested with $400 \mathrm{U} \mathrm{ml}^{-1}$ collagenase type III (Worthington Biochemical, Lakewood, NJ) at $37^{\circ} \mathrm{C}$ for 20 or $40 \mathrm{~min}$, respectively, and were ground between glass slides. Splenocytes were treated with red blood cell lysis buffer (Sigma-Aldrich) before suspension. Bone marrow (BM) cells were flushed from the femurs and tibias. Single-cell suspensions were obtained by forcing through a $40-\mu \mathrm{m}$ cell strainer (BD Biosciences, San Jose, CA). For the isolation of colonic LP leukocytes, ${ }^{31}$ large intestines were opened longitudinally, washed to remove fecal content, and cut into small pieces. To remove ECs, intestinal segments were treated in phosphate-buffered saline (PBS) containing $10 \%$ fetal calf serum (FCS), $20 \mathrm{~mm}$ HEPES, $100 \mathrm{U} \mathrm{ml}^{-1}$ penicillin, $100 \mu \mathrm{g} \mathrm{ml}^{-1}$ streptomycin, $1 \mathrm{~mm}$ sodium pyruvate, $20 \mathrm{~mm}$ EDTA, and $10 \mu \mathrm{g} \mathrm{ml}^{-1}$ polymyxin B (Calbiochem, Billerica, MA) with continuous stirring at $37^{\circ} \mathrm{C}$ for $20 \mathrm{~min}$ in a water bath. After wash with PBS, remaining tissues were incubated with $400 \mathrm{U} \mathrm{ml}^{-1}$ collagenase type III, $250 \mathrm{mU} \mathrm{ml}^{-1}$ dispase (Life Technologies, Carlsbad, CA), and $100 \mu \mathrm{g} \mathrm{ml}^{-1}$ DNase I (Roche Diagnostics, Basel, Switzerland) for $20-30 \mathrm{~min}$ at $37^{\circ} \mathrm{C}$ in a water bath. The cell suspension was prepared by forcing through a $100-\mu \mathrm{m}$ cell strainer, washed with PBS, resuspended in $10 \mathrm{ml}$ of $30 \%$ Percoll (GE Healthcare, Chicago, IL), and overlaid on $2 \mathrm{ml}$ of $70 \%$ percoll in a $15-\mathrm{ml}$ tube. Percoll gradient separation was performed by centrifugation at $780 \mathrm{~g}$ for $20 \mathrm{~min}$ at room temperature. The LP leukocytes were collected at the interface of the Percoll gradient and washed with RPMI-1640/10\% FCS, and used immediately for experiments. In some experiments,

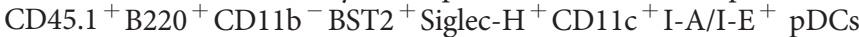
with $>99 \%$ purity were prepared by culturing BM cells obtained from B6.CD45.1 mice with human Flt3-L $\left(50 \mathrm{ng} \mathrm{ml}^{-1}\right.$, Wako Pure Chemical Industries, Osaka, Japan) for 8 days as described elsewhere followed by purification using a FACSAriaII cell sorter with fluorescein-conjugated monoclonal antibodies (mAbs; BD Biosciences, San Jose, CA).

Flow cytometry. Cells were stained with fluorescein-conjugated $\mathrm{mAbs}$ to mouse B7-H1 (MIH5), CD4 (RM4-5), CD8 $\alpha$ (53-6.7), CD11c (HL3), CD40 (3/23), CD45R/B220 (RA3-6B2), CD45.1 (A20), CD45.2 (104), CD69 (H1.2F3), CD80 (16-10A1), CD86 (GL1), IFN- $\gamma$ (XMG1.2), IL-17A (TC11-18H10), I-A/I-E (M5/114.15.2), Ly6C (HK1.4), Ly6G (1A8), isotype-matched control mAb (BD Biosciences), $\alpha 4 \beta 7$ (DATK32), B7-DC (TY25), B7-H2 (HK5.3; eBioscience, San Diego, CA), CCR9 (CW-1.2), CD3e (145-2C11), CCR2 (REA538), CD11b (M1/70), CD62L (MEL-14), CD64 (X54-5/ 7.1), CXCR3 (CXCR3-173), Foxp3 (FJK-16s), F4/80 (BM8), Siglec-H (551; Biolegend, San Diego, CA), mPDCA-1/BST2 (JF05-1C2.4.1), and Siglec-F (ES22-10D8; Miltenyi Biotec, Bergisch Gladbach, Germany). Intranuclear Foxp3 staining was performed as previously described. ${ }^{31}$ For the intracellular expression of cytokines, ${ }^{31}$ cells were incubated for $4 \mathrm{~h}$ with phorbol 12 -myristate 13 -acetate $\left(50 \mathrm{ng} \mathrm{ml}^{-1}\right.$; Sigma-Aldrich) and ionomycin (500 $\mathrm{ng} \mathrm{ml}^{-1}$; Sigma-Aldrich) plus GolgiPlug (BD Biosciences) during the final $2 \mathrm{~h}$. Subsequently, the cells were resuspended in Fixation-Permeabilization solution (eBioscience) and intracellular cytokine staining was carried out according to the manufacturer's directions. Fluorescence staining was analyzed with a FACSVerse flow cytometer (BD Biosciences) and FlowJo software (Tree star, Ashland, OR). In some experiments, flow cytometry analysis for the identification of leukocytes in the intestinal tissues was performed according to the previous reports ${ }^{27,28,44}$ with some modifications.

DSS-induced experimental colitis. Induction and evaluation of DSS-induced colitis were performed according to the previous reports ${ }^{26-28,45,46}$ with some modifications. In brief, mice were given 2\% DSS (molecular weight 36,000-50,000; ICN Biomedicals, Burlington, ON, Canada) dissolved in sterile distilled water for 7 days. BW, diarrhea (score: $0-3$ ), and bleeding (0-3) were monitored daily. DAI was determined as combined score of diarrhea and bleeding (0-6) on day 7 after the start of DSS treatment. The photographs of colon were taken, and their lengths were measured on day 7 after the start of DSS treatment. Similarly, mice were orally administrated with $2 \%$ DSS for 7 days, then switched to normal drinking water, and the disease progression, including changes in BW and survival time, was monitored for 14 days. In some experiments, CD $45.1^{+} \mathrm{B} 220^{+}$ $\mathrm{CD}_{11 \mathrm{~b}}{ }^{-}$pDCs prepared as described above $\left(5 \times 10^{6}\right.$ per mouse $)$ were 
intravenously injected into mice on 1 day before DSS treatment and day 3 after the start of the treatment. Alternatively, mice received daily i.p. injection of FTY720 ( $1 \mathrm{mg} \mathrm{kg}^{-1}$ per BW; Cayman chemical, Montigny-le-Bretonneux, France) from 1 day before DSS treatment to day 6 after the start of the treatment.

Culture of colon explants. Tissues from the middle colon obtained from untreated and DSS-treated mice on day 7 after the start of DSS treatment were isolated and rinsed in PBS, and cut into small segments $(\varphi 4 \mathrm{~mm})$ using disposable biopsy punches (Kai Industries, Tokyo, Japan). Colonic explants were cultured in 48-well tissue culture plates (BD Falcon, Franklin Lakes, NJ) in $500 \mu \mathrm{l}$ of RPMI medium-1640 at $37^{\circ} \mathrm{C}$ for $24 \mathrm{~h}$ in an atmosphere containing $5 \% \mathrm{CO}_{2}$. Culture supernatants were collected and stored at $-30^{\circ} \mathrm{C}$ until use.

Statistical analysis. Data are expressed as the mean \pm s.d. from 3 to 10 individual samples in a single experiment, and we performed at least three independent experiments. The statistical significance of the differences between the values obtained was evaluated by analysis of variance. A $P$ value of $<0.01$ was considered significant.

SUPPLEMENTARY MATERIAL is linked to the online version of the paper at http://www.nature.com/mi

\section{ACKNOWLEDGMENTS}

We thank all members of the animal facility at University of Miyazaki; Y. Sato for secretarial assistance; Y. Kawagoe for technical help in cell sorting; M. Aguet for providing Ifnar $1^{-1-}$ mice; and S. Jung for providing CD11c-DTR mice. This work was supported by Grants-in-aid for Scientific Research (B) (K.S.) and for Young Scientists (B) (H.T. and T.F.) from the Ministry of Education, Science and Culture of Japan, the Project for Cancer Research And Therapeutic Evolution (P-CREATE) from Japan Agency for Medical Research and Development (AMED) (K.S.), the Uehara Memorial Foundation (K.S. and H.T.), the Mochida Memorial Foundation for Medical and Pharmaceutical Research (K.S.), Takeda Science Foundation (H.T.), Kato Memorial Bioscience Foundation (H.T.), The NOVARTIS Foundation (Japan) for the Promotion of Science (H.T.), and Nagao Memorial Fund (H.T.). This work is licensed under a Creative Commons AttributionNonCommercial-NoDerivs 4.0 Inter-national License. The images or other third party material in this article are included in the article's Creative Commons license, unless indicated otherwise in the credit line; if the material is not included under the Creative Commons license, users will need to obtain permission from the license holder to reproduce the material. To view a copy of this license, visit http://creative-commons.org/ licenses/by-nc-nd/4.0/.

\section{AUTHOR CONTRIBUTIONS}

K.S. designed all experiments, analyzed the data, and wrote the manuscript; K.A., H.T., T.U., T.F., T.N. and N.C. performed the experiments; and Y.H. and Y.Y. provided reagents and information.

\section{DISCLOSURE}

The authors declare no conflict of interest.

Official journal of the Society for Mucosal Immunology

\section{REFERENCES}

1. Villadangos, J.A. \& Schnorrer, P. Intrinsic and cooperative antigenpresenting functions of dendritic-cell subsets in vivo. Nat. Rev. Immunol. 7, 543-555 (2007).

2. Watowich, S.S. \& Liu, Y.J. Mechanisms regulating dendritic cell specification and development. Immunol. Rev. 238, 76-92 (2010).

3. Guilliams, M. et al. Dendritic cells, monocytes and macrophages: a unified nomenclature based on ontogeny. Nat. Rev. Immunol. 14, 571-578 (2014).

4. Hildner, K. et al. Batf3 deficiency reveals a critical role for $\mathrm{CD} 8 \alpha^{+}$dendritic cells in cytotoxic T cell immunity. Science 322, 1097-1100 (2008).
5. Dresch, C., Leverrier, Y., Marvel, J. \& Shortman, K. Development of antigen cross-presentation capacity in dendritic cells. Trends Immunol. 33, 381-388 (2012).

6. Uto, T. et al. Clec4A4 is a regulatory receptor for dendritic cells that impairs inflammation and T-cell immunity. Nat. Commun. 7, 11273 (2016).

7. Gilliet, M., Cao, W. \& Liu, Y.J. Plasmacytoid dendritic cells: sensing nucleic acids in viral infection and autoimmune diseases. Nat. Rev. Immunol. 8, 594-606 (2008).

8. Swiecki, M. \& Colonna, M. Unraveling the functions of plasmacytoid dendritic cells during viral infections, autoimmunity, and tolerance. Immunol. Rev. 234, 142-162 (2010).

9. Reizis, B. Regulation of plasmacytoid dendritic cell development. Curr. Opin. Immunol. 22, 206-211 (2010).

10. Gilliet, M. \& Lande, R. Antimicrobial peptides and self-DNA in autoimmune skin inflammation. Curr. Opin. Immunol. 20, 401-407 (2008).

11. Glitzner, E. et al. Specific roles for dendritic cell subsets during initiation and progression of psoriasis. EMBO Mol. Med. 6, 1312-1327 (2014).

12. Sisirak, V. et al. Genetic evidence for the role of plasmacytoid dendritic cells in systemic lupus erythematosus. J. Exp. Med. 211, 1969-1976 (2014).

13. Rowland, S.L. et al. Early, transient depletion of plasmacytoid dendritic cells ameliorates autoimmunity in a lupus model. J. Exp. Med. 211, 1977-1991 (2014).

14. Davison, L.M. \& Jørgensen, T.N. SiglecH ${ }^{+}$plasmacytoid dendritic cells drive spontaneous lupus-like disease development in B6.Nba2 mice. Arthritis Rheumatol. 67, 1012-1022 (2015).

15. Ohno, H. Intestinal M cells. J. Biochem. 159, 151-160 (2016).

16. Lepage, P. et al. A metagenomic insight into our gut'smicrobiome. Gut 62 , 146-165 (2013).

17. Tsuji, N.M. \& Kosaka, A. Oral tolerance: intestinal homeostasis and antigen- specific regulatory T cells. Trends Immunol. 29, 532-540 (2008).

18. Fukaya, T. et al. Crucial roles of $\mathrm{B} 7-\mathrm{H} 1$ and $\mathrm{B} 7-\mathrm{DC}$ expressed on mesenteric lymph node dendritic cells in the generation of antigen-specific $\mathrm{CD}^{+}{ }^{+}$Foxp $^{+}{ }^{+}$regulatory $\mathrm{T}$ cells in the establishment of oral tolerance. Blood 116, 2266-2276 (2010).

19. Shanahan, F. Inflammatory bowel disease: immunodiagnostics, immunotherapeutics, and ecotherapeutics. Gastroenterology 120, 622-635 (2001).

20. Hooper, L.V. \& Gordon, J.I. Commensal host-bacterial relationships in the gut. Science 292, 1115-1118 (2001).

21. Podolsky, D.K. Inflammatory bowel disease. N. Engl. J. Med. 347, 417-429 (2002).

22. Round, J.L. \& Mazmanian, S.K. The gut microbiota shapes intestinal immune responses during health and disease. Nat. Rev. Immunol. 9, 313-323 (2009).

23. Strober, W., Fuss, I.J. \& Blumberg, R.S. The immunology of mucosal models of inflammation. Annu. Rev. Immunol. 20, 495-549 (2002).

24. Wirtz, S., Neufert, C., Weigmann, B. \& Neurath, M.F. Chemically induced mouse models of intestinal inflammation. Nat. Protoc. 2, 541-546 (2007).

25. Rakoff-Nahoum, S., Paglino, J., Eslami-Varzaneh, F., Edberg, S. \& Medzhitov, R. Recognition of commensal microflora by toll-like receptors is required for intestinal homeostasis. Cell 118, 229-241 (2004).

26. Abe, K. et al. Conventional dendritic cells regulate the outcome of colonic inflammation independently of T cells. Proc. Natl Acad. Sci. USA 104, 17022-17027 (2007).

27. Nakanishi, Y., Sato, T. \& Ohteki, T. Commensal Gram-positive bacteria initiates colitis by inducing monocyte/macrophage mobilization. Mucosal Immunol. 8, 152-160 (2015).

28. Asano, K. et al. Intestinal $\mathrm{CD}_{169}{ }^{+}$macrophages initiate mucosal inflammation by secreting CCL8 that recruits inflammatory monocytes. Nat. Commun. 21, 7802 (2015).

29. Baumgart, D.C. et al. Patients with active inflammatory bowel disease lack immature peripheral blood plasmacytoid and myeloiddendritic cells. Gut 54, 228-236 (2005).

30. Baumgart, D.C. et al. Aberrant plasmacytoid dendritic cell distribution and function in patients with Crohn's disease and ulcerative colitis. Clin. Exp. Immunol. 166, 46-54 (2011). 
31. Takagi, H. et al. Plasmacytoid dendritic cells are crucial for the initiation of inflammation and T cell immunity in vivo. Immunity 35, 958-971 (2011).

32. Takagi, H. et al. Plasmacytoid dendritic cells orchestrate TLR7-mediated innate and adaptive immunity for the initiation of autoimmune inflammation. Sci. Rep. 6, 24477 (2016).

33. Jung, S. et al. In vivo depletion of $\mathrm{CD} 11 \mathrm{C}^{+}$dendritic cells abrogates priming of $\mathrm{CD}^{+} \mathrm{T}$ cells by exogenous cell-associated antigens. Immunity 17, 211-220 (2002).

34. Müller, U. et al. Functional role of type I and type II interferons in antiviral defense. Science 264, 1918-1921 (1994).

35. Lombardi, V.C. \& Khaiboullina, S.F. Plasmacytoid dendritic cells of the gut: relevance to immunity and pathology. Clin. Immunol. 153, 165-177 (2014).

36. Gao, Y., Majchrzak-Kita, B., Fish, E.N. \& Gommerman, J.L. Dynamic accumulation of plasmacytoid dendritic cells in lymph nodes is regulated by interferon- $\beta$. Blood 114, 2623-2631 (2009).

37. Muzaki, A.R. etal. Intestinal $\mathrm{CD} 103^{+} \mathrm{CD} 11 \mathrm{~b}^{-}$dendritic cells restrain colitis via IFN- $\gamma$-induced anti-inflammatory response in epithelial cells. Mucosal Immunol. 9, 336-351 (2016).

38. Edelson, B.T. et al. Peripheral CD103 ${ }^{+}$dendritic cells form a unified subset developmentally related to $\mathrm{CD} 8 \alpha^{+}$conventional dendritic cells. J. Exp. Med. 207, 823-836 (2010).

39. Mizuno, S. et al. CCR9 ${ }^{+}$plasmacytoid dendritic cells in the small intestine suppress development of intestinal inflammation in mice. Immunol. Lett. 146, 64-69 (2012).

40. Tezuka, H. et al. Prominent role for plasmacytoid dendritic cells in mucosal T cell- independentole for plasmac. Immunity 34, 247-257 (2011).

41. González-Navajas, J.M., Lee, J., David, M. \& Raz, E. Immunomodulatory functions of type I interferons. Nat. Rev. Immunol. 12, 25-135 (2012).
42. Katakura, K. et al. Toll-like receptor 9-induced type I IFN protects mice from experimental colitis. J. Clin. Invest. 115, 695-702 (2005).

43. Rauch, I. et al. Type I interferons have opposing effects during the emergence and recovery phases of colitis. Eur. J. Immunol. 44, 2749-2760 (2014).

44. Tamoutounour, S. et al. CD64 distinguishes macrophages from dendritic cells in the gut and reveals the Th1-inducing role of mesenteric lymph node macrophages during colitis. Eur. J. Immunol. 42, 3150-3166 (2012).

45. Cooper, H.S., Murthy, S.N., Shah, R.S. \& Sedergran, D.J. Clinicopathologic study of dextran sulfate sodium experimental murine colitis. Lab. Invest. 69, 238-249 (1993).

46. Dieleman, L.A. et al. Chronic experimental colitis induced by dextran sulphate sodium (DSS) is characterized by Th1 and Th2 cytokines. Clin. Exp. Immunol. 114, 385-391 (1998).

This work is licensed under a Creative Commons Attribution 4.0 International License. The images or other third party material in this articleareincluded in thearticle's Creative Commons license, unless indicated otherwise in the credit line; if the material is not included under the Creative Commons license, users will need to obtain permission from the license holder to reproduce the material. To view a copy of this license, visit http://creativecommons.org/licenses/by/4.0/

(C) The Author(s) 2017 